\title{
DIRECCIÓN DE JUNTAS Y COMISIONES DE IGLESIA
}

Rojas Yauri, Benjamin Universidad Peruana Unión

\author{
benjamin@upeu.edu.pe
}

Fecha de recepción: Julio 2013

Fecha de aceptación y versión final: Noviembre 2013

El presente artículo aborda una parte importante del quehacer administrativo en la Iglesia, las reuniones llamadas juntas o comisiones, donde los directivos toman las decisiones que permiten a la Iglesia cumplir con sus objetivos y su razón de ser. Luego de explicar la importancia y el valor de estas reuniones, se pasa a explicar algunas técnicas y principios que, puestos en práctica, harán de las mismas herramientas útiles para la administración de la Iglesia. El documento concluye afirmando que el éxito de una iglesia estriba en contar con un método definido para efectuar juntas eficientes, que den gloria a Dios al cumplir sus dos mayores razones de ser y al practicar una correcta mayordomía en su ejecución.

Palabras clave: Administración, juntas, comisiones, iglesia. 


\section{LEADING CHURCH MEETINGS (COUNCILS) AND COMMITEES}

Summary: This article speaks of an important administrative duty in church, the meetings known as the councils, where the leaders make the decisions that allow the church to accomplish its objectives and address the reasons for its existence. After explaining the importance and the value of these meetings, we can explain some techniques and principles that if put in effect, will make of these instruments useful for church administration. The document concludes affirming that the success of a church strides in counting with a defined method to execute efficient assemblies that give glory to God in reaching its two major reasons of existence and in practicing a correct stewardship in its execution.

Keywords: Administration, Meetings, Assemblies, Comisions-Comitees, Church. 


\section{Introducción}

Las Juntas de Iglesia, coexistieron con la Iglesia, no es necesario recorrer muchas páginas en el Nuevo Testamento para leer sobre juntas -concilios- en la Biblia. La práctica era común en el pueblo judío y el cristianismo, nacido en su seno, siguió el ejemplo y tuvo su primer concilio hacia el año 50 d.C.

La Iglesia Adventista del $7^{\circ}$ Día, sigue el legado de los discípulos, pero comprende que ella no es la razón de ser de la Iglesia, 124 sino que es un medio e instrumento que ayuda a su quehacer.

\section{Importancia y valor}

Aunque se reconoce que ningún pastor fue llamado por Dios para dedicarse a la tarea exclusiva de dirigir juntas de iglesia (función administrativa), se entiende que esa es una de sus responsabilidades. Sin embargo, al cumplir esta función debe recordar lo recordar lo escrito por Elena G. de White al respecto:
Un ministro no puede mantener su mente en la mejor condición espiritual mientras sea llamado a solucionar pequeñas dificultades en las diversas iglesias. No es esta la obra que se le ha encomendado. Dios desea utilizar todas las facultades de sus mensajeros escogidos. Su mente no debería ser recargada por largas juntas realizadas en la noche, porque Dios desea que toda su capacidad mental sea utilizada en la proclamación con claridad y fuerza del Evangelio, tal como fue enseñado por Jesús. ${ }^{1}$

Esta cita no solo advierte al pastor sobre juntas inadecuadas, sino que también nos ayuda a ver su importancia y valor. Pues se nos dice que una junta solo es importante y de valor ante los ojos de Dios, si ella usa el tiempo de forma correcta -es decir, si no se pierde el tiempo en charlas sin sentido, poco espirituales y muchas veces aun perjudiciales para la Iglesia de Dios-, pues el tiempo es uno de los dones más valiosos que el Señor nos ha dado.

1 Elena G. de White, El evangelismo (Buenos Aires: Casa Editora Sudamericana, 1991), 72. 
De forma puntual se puede decir que una junta de iglesia tiene importancia y valor si: 1 ) soluciona grandes dificultades de la iglesia, y 2) si sirve para el quehacer evangelístico de la iglesia. Empero, sean dos simples oraciones, la ejecución de las mismas es un gran desafío, pues el pastor debe ser sabio para evaluar cuáles son las grandes dificultades de la Iglesia, y obediente ${ }^{2}$ para cumplir con estos dos objetivos o razones de ser de la Junta de Iglesia. Si se cumplen estas declaraciones, entonces se podrá decir que esa junta y su pastor honran a Dios, pero si no lo hacen, entonces Él pedirá cuentas por el tiempo mal invertido y por haber perjudicado a la iglesia al no haber alcanzado los dos grandes objetivos de toda Junta de Iglesia.

2 Se dice obediente, ya que el consejo del Manual de la Iglesia respecto a la agenda de la Junta de Iglesia dice que "El primer punto de la agenda de cada reunión de la Junta Directiva de la iglesia debe ser planificar la evangelización del territorio misionero de la Iglesia”. Asociación General de la Iglesia Adventista del $7^{\circ}$ Día, Manual de la Iglesia, trad., Roberto Gullón, 6ta ed. (Buenos Aires: Asociación Casa Editora Sudamericana, 2011), 126.

\section{Procedimiento a seguir en las reuniones}

La forma de las reuniones puede variar de un lugar a otro en ciertos aspectos, ya que son los voluntarios que componen la Junta de Iglesia quienes decidirán algunas normas, reglas y costumbres respecto a esta importante reunión. Sin embargo, el pastor de iglesia debe guiar, corregir u orientar a los feligreses en el establecimiento de ellas. Cuando se dice la forma de la reunión, se habla de aspectos como: horarios, lugar, tiempo, participantes, sistema de convocatoria, sistema de votación, etc.

Sin embargo, el procedimiento a seguir, en las juntas, deberá ser lo más parecido posible en todas las iglesias, pues ella es el fondo de la junta. Aquí nos referimos a: la agenda, el sistema de registro, la dirección, intervenciones y reintervenciones, entre algunas. Algunos consejos puntuales respecto al procedimiento a seguir para llevar a cabo una junta o comisión son: 
1. Nunca una junta debe ser convocada sin el conocimiento del pastor de iglesia, (Solo el presidente del campo local puede convocar a una junta, pero, por ética, esta deberá ser coordinada con el pastor distrital).

2. Vote, en la primera junta o comisión, las normas, reglas y costumbres que regirán sus reuniones [horario, lugar, quórum, tiempo (de la junta y de las participaciones), sistema de votación, intervenciones, segunda intervención, inicio y cierre, etc.].

3. Asegúrese de contar con un quórum posible y que no entorpezca las reuniones de las juntas o comisiones. Es importante en este sentido seguir el modelo de la iglesias grandes como la de Villa Unión, donde el quórum es de 7 , aun cuando los miembros de la junta son más de 50.
4. Tenga la agenda preparada con anticipación. La agenda no debe ser improvisada, debe ser elaborada por la secretaría de la iglesia. Si la secretaría de la iglesia no tiene la experiencia suficiente en el cargo, debe ser ayudada/o por el pastor o un anciano de experiencia.

5. Facilite de forma visible el orden del día - la agenda -, este debe incluir las metas y objetivos de esa junta o comisión.

\section{Principios para la dirección}

Cuando se habla de juntas y comisiones, lo más importante es conocer los principios necesarios para realizar una buena dirección de las mismas, estos nos ayudarán a ser eficientes pero sobre todo a cumplir con las dos razones de ser de toda junta o comisión de la iglesia. Sin embargo, sobre toda las cosas, el dirigente de iglesia debe 
tener un genuino conocimiento de la Palabra de Dios, debe confiar en ella y debe vivir en base a ella, Elena G. de White dice al respecto:

Deben ser hombres de perfecta integridad, no novicios, sino estudiantes inteligentes de la Palabra, capaces también de enseñar a otros, sacando de su tesoro cosas nuevas y viejas; hombres que, en su carácter, palabras y porte, honren la causa de Cristo, enseñando la verdad y viviendo en conformidad con ella, desarrollándose hasta alcanzar la plena estatura en Cristo Jesús. ${ }^{3}$

Si bien es cierto alguien podría decir con razón, que el conocimiento profundo de la Palabra de Dios es suficiente para ser un dirigente eficiente de la Iglesia de Dios, es necesario que se evalúen algunos otros puntos más.

3 Elena G. de White, Obreros evangélicos (Buenos Aires: Casa Editora Sudamericana, 1991), 427.

\section{Conocimiento del sistema administrativo de la Iglesia}

En este sentido es importante que todo líder de iglesia tenga en su poder y conozca -es decir los haya leído en más de una oportunidad-, el contenido de los siguientes materiales como mínimo: 1) Manual de Iglesia, 2) Guía de procedimiento para ancianos, 3) Guía de procedimiento para ministros.

Además de estos documentos oficiales de la Iglesia adventista mundial, se puede hacer uso y es necesario conocer documentos emitidos por la división, unión o campo local. También existen otros materiales de lectura importantes sobre este tema. ${ }^{4}$

4 Véase los siguientes materiales: Jonas Arrais, Una iglesia positivia en un mundo negativo: aprendiendo y mejorando el liderazgo en cada experiencia de tu iglesia (Buenos Aires: Asociación Casa Editora Sudamericana, 2008); Richard Exley, Peligros del poder (Idaho: Pacific Press Publishing Association, 1998); Roberto H. Pierson, Para usted que quiere ser dirigente (Idaho: Pacific Press Publishing Association, 1998); John Rhodes, Secretos del éxito para pastores (Mariland: Review and Herald Graphics, 1998); Elena G. de White, El ministerio pastoral (Maryland: Asociación 


\section{Conocimientos sobre liderazgo eficiente}

Algunos principios puntuales para la dirección de Jutas son los siguientes:

1. Sea creativo, no sea aburrido, "En la administración eclesiástica es posible mejorar muchas de nuestras actividades programas y tareas empleando la creatividad". 5

2. Supere su temor al cambio, recuerde que "No es cambio o que estemos tan enamorados de las formas antiguas. Es al espacio entre medio de los dos al que le tenemos". ${ }^{6}$

3. Use palabras positivas recuerde que "las palabras positivas facilitan las relaciones humanas y crean una atmósfera pacífica, que provee des-

Ministerial de la Asociación General de los Adventistas del Séptimo Día, 1997).

5 Arrais, 19.

6 Ibid., 37.

canso, naturalidad, rejuvenecimiento y sueño, todos necesarios para la salud". ? Por ejemplo frente a la elección de un nuevo anciano, pregunte si alguien tiene algo positivo para decir sobre los nombres propuestos, no permita que alguien diga algo negativo.

4. No viole el principio de la confidencialidad, ni motive a que alguien lo haga por ningún motivo [por ejemplo el tesorero al hablar de aquellos que diezman o no], recuerde que "cuando se rompen las confidencias, se pueden producir daños relacionales, emocionales, vocacionales e incluso espirituales". ${ }^{8}$

5. Haga todo con transparencia y del modo correcto, de forma que se note que Ud. es un líder cuya motivación de su accionar es el servicio, esto

7 Ibid., 73.

8 Ibid., 97,98. 
aun si se trata de una reunión administrativa en la que se tenga que disciplinar a un feligrés.

6. Trabaje en un ambiente de igualdad, pues en la obra de Dios no existen subordinados "Todos trabajamos juntos en la misma obra de Dios. Trabajamos unos con otros. Algunos tienen distritos o parroquias más grandes que otros".

7. Sea empático en su liderazgo, recuerde que si "podemos participar de la experiencia de nuestros colaboradores, andaremos por el camino del verdadero liderazgo cristiano". 10

8. Maneje sabiamente la oposición, pero no use el método Lincoln quien, en cierta oportunidad, en su parlamento luego de una votación en la que quedó solo dijo "siete no, y un sí. Gana el sí”.

9 Pierson, 41.

10 Ibid., 64.

\section{Aspectos puntuales en la dirección de juntas y comisiones}

Pierson señala algunas cosas puntuales que un líder debe hacer cuando Dios le ha dado la oportunidad de dirigir una junta: 1) Permita que los miembros de la junta expresen sus opiniones, 2) Sepa cuándo cristalizar el pensamiento de su junta, es decir, no permita que los miembros de junta den vuelta sobre un asunto sin fin, 3) Postergue, cambie o anule sus planes y opiniones si es necesario, 4) Medie y negocie de tal modo que al repartir la torta todos crean que recibieron la porción más grande, 5) Cumpla con los acuerdos de junta, 6) Antes de tomar una decisión, asegúrese de que $\mathrm{Ud}$. y los miembros de su junta o comisión tengan toda la información necesaria sobre el tema, 7) Distribuya, ahorre y use de forma sabia el tiempo. ${ }^{11}$

11 Elena G. de White dice al respecto "Se nos amonesta a redimir el tiempo. Pero el tiempo desperdiciado no puede recuperarse jamás. No podemos hacer retroceder ni un solo momento. La única manera en 


\section{Modelo de reunión de comisión o junta}

1. Inicie la reunión con una oración, dependiendo de la situación puede haber un canto y una lectura bíblica.

2. Presente la agenda de la reunión y explique los objetivos y metas de la reunión.

3. Desarrolle los dos primeros puntos que nunca deben faltar en una segunda reunión de cualquier junta o comisión (estos puntos también pueden ser desarrollados en una primera reunión, pero no siempre es posible)

a. Lectura del acta última de esta comisión o junta. El propósito de este punto es eva-

la cual podemos redimir nuestro tiempo es aprovechando lo más posible el que nos queda, colaborando con Dios en su gran plan de redención". Elena G. de White, Palabras de vida del gran maestro (Buenos Aires: Casa Editora Sudamericana, 1991), 277.

luar el cumplimiento de los votos tomados.

b. El quehacer evangelístico de la iglesia debe ser evaluado, se debe orar por ello y se debe votar en el caso de las comisiones, cómo se contribuirá en el cumplimiento de la misión. En el caso de la junta de iglesia el director de MIPES puede presentar un corto informe en este momento a la junta.

4. Continúe presentando los otros puntos de agenda, uno a la vez y sigua el siguiente modelo.

a. Presentación del punto de agenda: El secretario/a de iglesia lee el punto de agenda [Ej. Punto N. ${ }^{\circ} 2011$ 034 Compra de TV]

b. Pedido de propuestas: i. Pida una propuesta. Pregunte, ¿alguien propone 
que se compre el televisor? Si nadie propone, Ud. puede proponerlo y continuar con el siguiente paso.

ii. Pida observaciones. Recuerde que las observaciones deben ser positivas. La ausencia de observaciones indica que hay un desacuerdo general con este punto. iii. Pida otra propuesta, siga la indicación del inciso a y b para esta nueva observación. Se puede continuar pidiendo propuestas, lo recomendable es no pasar de tres propuestas.

c. Lleve las propuesta a votación, recuerde que siempre será la mayoría la que gane y por ningún motivo se debe manipular la votación de la junta o comisión de iglesia.

5. Repase todos los puntos votados en esa reunión, para ello la secretaría de la iglesia puede dar una lectura breve de sus apuntes (no es necesario que se lean todos los detalles).

6. Termine la sesión con una oración y dependiendo de lo acordado como parte de la forma de reunión para esa iglesia, se puede cantar o realizar otra actividad.

\section{Conclusiones}

Una herramienta vital para la buena administración de la iglesia estriba en contar con un método definido para efectuar juntas eficientes, este método debe ayudar a: 1) administrar correctamente el tiempo, 2) mantener la confidencialidad, 3) fomentar un espíritu de participación cordial, 4) agilizar el proceso de toma de decisiones, 5) solucionar los problemas de 
la iglesia de forma eficiente $y$ rápida y 6) cumplir el quehacer evangelístico de la iglesia.

Además de los mencionados, existen otros múltiples beneficios cuando se llevan a cabo juntas eficientes, mantiene a la iglesia en el sendero correcto, reduce el consumo de tiempo y energía y sobre todo incrementa la probabilidad de éxito en cada uno de los proyectos de iglesia.

Debido a la mencionado es imperioso que el pastor adventista esté cabalmente preparado

132 para llevar a cabo juntas eficientes, efectivas y que, sobre todo, den gloria a Dios al cumplir las dos mayores razones de ser de la juntas y comisiones de iglesia y al practicar una correcta mayordomía en su ejecución.

\section{Bibliografía}

Arrais, Jonas. Una iglesia positivia en un mundo negativo: aprendiendo y mejorando el liderazgo en cada experiencia de tu iglesia. Buenos Aires: Asociación Casa Editora Sudamericana, 2008.

Asociación General de la Iglesia Adventista del $7^{\circ}$ Día. Manual de la iglesia. Traducido por Roberto Gullón. 6ta ed. Buenos Aires: Asociación Casa Editora Sudamericana, 2011.

Exley, Richard. Peligros del poder. Idaho: Pacific Press Publishing Association, 1998.

Pierson, Roberto H. Para usted que quiere ser dirigente. Idaho: Pacific Press $\mathrm{Pu}-$ blishing Association, 1998.

Rhodes, John. Secretos del éxito para pastores. Mariland: Review and Herald Graphics, 1998.

White, Elena G. de. El evangelismo. Buenos Aires: Casa Editora Sudamericana, 1991.

White, Elena G. de. Obreros evangélicos. Buenos Aires: Casa Editora Sudamericana, 1991.

White, Elena G. de. Palabras de vida del gran maestro. Buenos Aires: Casa Editora Sudamericana, 1991.

White, Elena G. de. El ministerio pastoral. Maryland: Asociación Ministerial de la Asociación General de los Adventistas del Séptimo Día, 1997. 